\title{
An all-to-all approach to the identification of sequence-specific readers for epigenetic DNA modifications on cytosine
}

Guang Song ${ }^{1,6}$, Guohua Wang 2,6 , Ximei Luo $2,3,6$, Ying Cheng ${ }^{4}$, Qifeng Song ${ }^{1}$, Jun Wan ${ }^{3}$, Cedric Moore ${ }^{1}$, Hongjun Song ${ }^{5}$, Peng Jin ${ }^{4}$, Jiang Qian ${ }^{3, *}$, Heng Zhu ${ }^{1, *}$

The authors have withdrawn their manuscript whilst they perform additional experiments to test some of their conclusions further. Therefore, the authors do not wish this work to be cited as reference for the project. If you have any questions, please contact the corresponding author. 